\title{
Life beyond cancer: sexuality and fertility in cancer survivors
}

\author{
Francesca Romito \\ National Cancer Research Center “Giovani Paolo II”, Bari, Italy
}

Psychoonkologia 2015, 3: 83-87

DOI: $10.5114 /$ pson.2015.57167

\author{
Address for correspondence: \\ Francesca Romito \\ Psychologist, Psychotherapist \\ National Cancer Research Center \\ "Giovani Paolo II" \\ Bari, Italy \\ e-mail: francescaromito@yahoo.com
}

\begin{abstract}
Due to progress in cancer diagnosis and cancer therapies, the population of cancer survivors in Europe is rapidly growing. Cancer survivors have multiple psycho-social needs: loss of fertility and problems in sexuality are two major concerns that, if not adequately treated, may negatively impact survivors' psychological well-being and prevent the fulfillment of their existential plans, both at the individual and at the interpersonal level. Information and communication regarding these aspects are often neglected in the clinical practice whereas they must be addressed in a regular and systematic manner, and strategies to enhance communication and improve the provision of information should be implemented.
\end{abstract}

Key words: cancer survival, fertility, sexuality.

\section{Introduction}

Data from European cancer registries report that 5 -year survival rates of cancer patients ranges between $57 \%$ and $82 \%$ according to age, country and tumour site, with higher survival rates in breast, prostate and colorectal cancers [1]. The growing number of cancer survivors in Europe poses new challenges to the National Health Systems of European countries since it results in a growing number of "rehabilitation" needs that have to be addressed for improving cancer survivors' quality of life: all the psychological, physical and social challenges related to coming back to life. There are several domains of quality of life that may be potentially influenced by cancer diagnosis or cancer treatments, during the survival phase: anxiety and depression, cardiac toxicity, cognitive dysfunction, fatigue, pain, sexual dysfunctions, infertility and sleep disorders [2]. The ones that pose major strains on the relational life of young cancer survivors include several changes in sexual functioning and in fertility.

\section{Sexuality in cancer survivors}

The dimension of the problem of sexuality varies a lot according to aspects such as the cancer site, age at diagnosis, stage of the illness, time elapsed from treatments. All these aspects affect the prevalence and the level of impairment in sexual functioning in both sexes. The typology of sexual problems in women may include: reduced sexual desire and arousal, difficulty in reaching an orgasm, pain during intercourses, reduction in vaginal lubrication, alteration in body image, reduced sensitivity in breast and nipple. Prevalence of sexual problems in women may range from $30-100 \%$ in breast cancer to $6-60 \%$ in colorectal cancer [3]. Men may suffer from erectile dysfunction or impotence that may include problems in sexual desire, orgasm or ejaculation, and may not necessarily be linked with reaching or maintaining an erection. The prevalence of erectile dysfunction in men with prostate cancer may vary from $14 \%$ to $90 \%$, of the overall sexual difficulties from $39 \%$ to $50 \%$ in those suffering from colorectal cancer [3].

To understand the complex model of factors affecting sexuality, we do rely on the bio-psychosocial model of health [4]: several domains are connected to cancer-related sexual problems and when performing an assessment of cancer survivors we should take into account a variety of biologic, psychological, interpersonal and social/cultural aspects. 


\section{Biology}

I didn't really realize the radiation would affect my sexuality until it happened. I don't think anyone can tell you what the pain discomfort and exhaustion will do to you. (Woman, 61 years old, hetero, digestive/gastrointestinal cancer $)^{1}$

When examining the biologic domain, with respect to women, hormonal treatments (e.g. aromatase inhibitors) may increase menopausal symptoms, causing hot flashes or vaginal dryness; the irradiation of the pelvis may predispose to vaginal fibrosis, stenosis and loss of lubrication, causing pain during intercourses; moreover, general changes in body appearance, such as loss of weight or excessive weight gain, hair loss, scarring, may alter body integrity and body image [3]. Regarding men, prostate cancer, despite the improvement in surgical techniques, frequently leads to erectile dysfunction, and androgen deprivation therapy is associated with gynecomastia, hot flashes, emotional lability, which may undermine masculine identity [3]. For both men and women, a reduced sensitivity in the body, weakness, fatigue and pain may negatively impair the possibility of fully enjoying sexual life. Both male and female patients who undergo bone marrow transplantation may have severe genital graft-versus-host disease even many years after treatment, with pain, inflammation, stenosis (for women) and curvature of the penis (for men) [3].

\section{Psychology and emotions}

I just don't want to have her touch me because I don't feel I deserve it or feel worthwhile. (Woman, 45 years old, lesbian, breast cancer)

The psychological and emotional aspects of cancer and survival are directly related to sexual problems: depression, anxiety, worries, uncertainty about the future alter survivors' mood; the same happens with negative cognitions or dispositions such as helplessness and hopelessness. The alterations in body image may increase the feeling of being unattractive, and this is particularly important to women since this seems to prevent them from engaging in sexual activity [5].

\section{Interpersonal aspects}

I find since my wife has been reluctant to provide physical support as and when I would like it, this in itself has put a great strain on our sexual relationship. (Man, 57 years old, hetero, prostate cancer)
To what concerns the interpersonal domain, this is the one where all the relational, family and couple issues are strictly interdependent. There is a huge amount of literature on couple changes after the cancer experience [e.g. 6]: cancer poses major strains on relationships and their previous balance. The cancer experience may work as a testbed of the strength of significant relationships, a time to verify the quality of the bonds. During time of need, like the illness, often the patient verifies, as on the Boszormenyi-Nagy "account book" [7], whether he/she is receiving the expected support from significant others. An important Italian family therapist, Maurizio Andolfi, wrote: "Most of the couples spend years on trying not to reveal each other as they are for real, only in order to maintain an illusion of a complete union, thereby suffocating any possible real affective connection" [8]. The illness may have the risk of working as a detonator of pre-existing conflicts that have been silent for many years. Thus, the significant relationships always get out modified or at least "verified" after the cancer experience: the survivor may have experienced lack of support from the partner and this awareness works like a wound in the couple relationship, negatively affecting love, affection and sex. Or the problem may be more specifically related to sex, with the assumption that sexual life has just ended, with no member of the couple openly discussing the problem, perpetuating problems and living "with an elephant in the room" [3].

There are also social and cultural aspects that are strictly interrelated with sexual activity or rehabilitation: just to make an example, vaginal dilators are among the most effective treatment methods for vaginal stenosis after radiotherapy in the pelvis [9]. This tool may be particularly embarrassing for women who do not feel confident with self-touch. Other sexual rehabilitation strategies may include self-stimulation for delivering oxygenated blood flow to genital tissue [9], but also this recommendation may be complicated by personal and cultural beliefs about masturbation. This aspect may also rely in the minds of care-providers with some prejudices or false assumptions regarding sex, such as that it is not an important issue for widowers, or single or older individuals. Aspects such as "different" sexual orientation or sexual identity may add a further filter in the already difficult communication around these aspects.

An interesting study has investigated the survivors' subjective experience of changes in sexual well-being using quantitative and qualitative 
methods. Ussher and colleagues [5] have interviewed six hundred and fifty seven cancer survivors to understand the complex interactions of various causes of sexual problems. They found a significant reduction both in sexual frequency and in sexual satisfaction after cancer both in men and women. As regards to sexual frequency, $12 \%$ of women and $13 \%$ of men reported sex occurring never or rarely before the cancer diagnosis, compared to $52 \%$ of women and $41 \%$ of men making the same report after the experience of cancer. As concerns sexual satisfaction, both men and women rated their sexual relationship as significantly less satisfying after cancer, with $48.8 \%$ of women and $44.4 \%$ of men rating their current relationship as unsatisfying, compared to $6.7 \%$ of women and $4.3 \%$ of men before having had the cancer. This significant reduction in frequency and satisfaction was found in all cancer survivors, independent of age, cancer type, time passed from diagnosis and duration of relationship. Survivors with cancer in reproductive organs (breast, prostate or gynecologic) were significantly more likely to report dissatisfaction in frequency and quality of intercourses compared to those with a non-reproductive cancer. Not only were the sexual intercourses diminished after the illness, but also a variety of sex-related behaviors: kissing, petting, self or partner-masturbation, oral sex and the use of sex toys (exception made for men who use more sex toys after the cancer). The authors addressed the point of perceived causes at the basis of the observed changes in sexual activity. The "top" three causes in women were: sex-specific difficulties (vaginal dryness), tiredness and feeling unattractive. Men rated the following as the "top" three: sex-specific difficulties (erectile dysfunction), getting older and surgery.

\section{Communication}

One of the most significant barriers to adequately address sexual problems is the lack of communication around this issue. Several years ago, the Journal of Clinical Oncology published a paper entitled: "The Sound of Silence: sexuality information for cancer patients" [10], claiming a loss of exchange among doctors and patients regarding sexual problems and sexual rehabilitation strategies. It was 2005 , and unfortunately in this decade probably things have not changed so much. Physicians are often reluctant to raise this topic, probably due to time constraints and lack of preparation, or also because of the paucity of available multidisciplinary resources to offer specific counseling to patients. Patricia Ganz, an oncologist very committed to cancer survivors, one of the researchers who has stimulated many studies on cancer survival in the U.S., in an interview admitted that clinicians have a "don't ask, don't tell" policy, when dealing with sexual health [11]. At the same time, patients do not feel confident to talk for first about this problem, they may feel embarrassed or ashamed. In this way, the silence around this issue has the negative consequence to confirm the already erroneous assumptions of survivors, like the one that sexual problems are simply among the treatment-related side effects "and nothing can be done about it".

Website personalized information, booklets or some very short distress screening methods may be a first-line effective way to start to shed light on such a "forgotten" issue and start to raise the argument among patients and oncologists during follow-up visits.

\section{Fertility}

Even if cancer is more frequent in the older age, it also affects children, adolescents and young adults, and survival rates are luckily the highest among patients under 45 years old [1]. Cancer itself is rarely a direct cause of infertility; cancer therapies, including chemotherapy, radiotherapy, hormonal therapy or surgery may cause infertility as a frequent side effect. In women, many chemotherapeutic drugs are toxic for the gonads, and alkylating agents offer the major risk of permanent infertility. Ionizing radiations are extremely toxic for oocytes and cranial radiation, which affect the hypothalamic-pituitary axis, is also a risk factor for infertility. Male germ cells are also sensitive to cytotoxic drugs [12].

There are some well-established methods to preserve fertility: the most approved ones are sperm cryopreservation for men; for women: embryo and oocyte cryopreservation and ovarian transposition. Sperm cryopreservation is possible usually by freezing three samples of sperm with at least 48 hours of abstinence between each collection, but also less samples may be collected if the treatment must be started urgently. A birth with a sperm frozen twenty-one years before has been described in the literature [13]. Embryo cryopreservation is not allowed in all countries, it delays treatment for two or three weeks, according to the menstrual cycle of the woman, and requires also the presence of a partner. Oocyte cryopreservation is allowed in all countries, and it also delays the beginning of treatment. Both procedures require an ovarian stimulation and there is a concern that it may increase cancer recurrence in estrogen-dependent tumors [14]. Oocyte cryopreservation is no more experimental since 2013 and up to now more than 900 births 
have been reported from cryopreserved oocytes [15]. Ovarian transposition consists in the surgical translocation of ovaries from the irradiation field in the pelvic area: but this method does not protect from chemotherapy or whole-body irradiation.

Infertility may affect up to $80 \%$ of cancer survivors [12]. There is a social aspect that complicates the problem: pregnancies in women older than 35 years are increasing; from $12 \%$ in 1990 to $16 \%$ in 1996 up to an estimation of $25 \%$ in 2025 [16]. With this trend, the problem of loss of parenthood in cancer survivors will surely increase in the following years.

\section{Communication}

As for sexuality, also for what concerns fertility, one of the most important barrier to the access to information and fertility options is the loss of communication. In a recent paper, the research group led by Fedro Peccatori at the European Institute of Oncology wrote: "Despite clinical and psychological benefits, and recommendations that cancer patients should be routinely asked about their interest to preserve fertility before starting cancer treatment, nearly half of patients are not given information about the impact of cancer treatment on their future fertility" [12]. British and American doctors seem to open the discussion on fertility on most occasions, while in most European countries this tendency is not so high and fertility consultations do not occur on a regular basis, although all international guidelines make recommendations in this respect [12].

For example, in 2012, a Swedish study has collected interviews of 484 survivors who have had cancer when they were 18-45 years old, and they reported that although $80 \%$ of male patients had information, only $48 \%$ of female patients were informed about risks concerning reproduction [17]. A German study found that $60 \%$ of young cancer survivors had discussed fertility with their doctors and only $20 \%$ had the opportunity to talk to a specialist [18]. Another German study of 2011 reported an increasing trend in communication over years: $67 \%$ of survivors diagnosed among 1980 and 1984 did not receive information on fertility, the same lack of communication applied to $50 \%$ of patients diagnosed in 2000-2004 [19].

These data are embarrassing and still worrying: the lack of information generates impairment and fears in cancer survivors. Even if they may be actually able to conceive, survivors may be afraid that pregnancy can increase the risk of cancer recurrence or to transmit the cancer to the offspring. Studies also found that survivors who reported a lack of counseling had offspring-related fears more frequently, which stopped them from having children [20].

Which kind of barriers may limit communication on fertility matters? A general tendency reported in most studies [12] is that physicians may be conditioned by the type of cancer, or by patients' prognosis and be worried to delay the start of treatment, or also they may not be completely aware of fertility preservation options. Other prejudices that bias the communication on fertility may be linked to patients' socio-economic status or age, the presence of a partnered relationship, or of other children, of psychological aspects such as anxiety or the patient's sexual orientation. Unfortunately, also the female gender has been shown to expose patients to a major risk of not being told about the fertility risk and not actually use fertility preservation methods. These kind of biases that lie in oncologists' thoughts and behaviors, limit the patients' right to choose themselves for their future.

What do patients want and what do they choose when they can make a choice? Regardless of the final decision to actually adopt procedures for preserving fertility, the vast majority of patients consider fertility consultations as an important part of the treatment [20]. In the previously cited German study, $40 \%$ of patients interested in having children in the future, actually choose some fertility preservation options [18]. Data from a larger Swedish studies reveal that, whereas $56 \%$ of men choose to bank sperm, only $2 \%$ of women choose to rely on fertility preservation options [17]. A systematic review on attitudes towards fertility in various geographical and cultural groups found that $59 \%$ of cancer patients wanted to have children in the future but less than $10 \%$ would accept lower survival chances to preserve fertility [20]. However this study has also underlined a positive effect of having a child as a fundamental step of the complete recovery process.

The problem of lack of information and communication is a major one when planning opportune pathways for rehabilitation (in the case of fertility, it may be called pre-habilitation). The sixth Italian report on the supportive care conditions of cancer patients [21] produced by the Federation of Voluntary Associations in Oncology (FAVO) in 2014, proposes a future plan of "Objective: zero percent", hoping that, with adequate initiatives, the future percentage of patients without an appropriate counseling on fertility will be zero. One of the problems of how to accomplish this goal is related to the very rare coexistence in the same hospital settings of both oncology unit and unit of reproductive medicine which are competent for collection and conservation of biologic material. The system should work 
as a "hub and spoke" mechanism where most specialized centers of reproductive medicine are the "hub" that can offer expertise for complicated procedures or correct stocking of biologic materials or surgical procedures, whereas the other units (e.g. the oncology units) may refer to these centers as "spokes".

Access to information should be enhanced also with adequate training for doctors and other health professionals, to widespread the culture of the attention to fertility preservation in cancer patients. Patients should have easy access to this information, firstly on the drug information leaflets, where usually every single possible side effect is explained, but information on risk for fertility is often lacking. Secondly, it would be helpful to design educational tools such as information booklets, or websites where it is possible to obtain information and read about other people's experiences. Two examples of interesting web-based resources where patients can navigate to approach the problem of fertility are: www.myoncofertility.org, designed by the Oncofertility Consortium at the Northwestern University in the US and the Fertility Services of the Livestrong Association (www.livestrong.org/ we-can-help/fertility-services).

\section{Conclusions}

The population of cancer survivors in Europe is rapidly growing, and survivors have multiple needs and it is necessary to address them and rehabilitate from the early phases of the illness. Fertility and sexuality are two major concerns that, if not adequately treated, may have a strong impact on survivors' psychological well-being and prevent the fulfillment of their personal and interpersonal existential plans. These two aspects unfortunately share a common negative destiny: they are often neglected in the clinical practice thus generating problems in the future and present quality of life of cancer patients and survivors. Both fertility and sexuality must be addressed in a regular and systematic manner, and strategies to enhance communication and improve the provision of information should be implemented.

\section{Disclosure}

Author reports no conflict of interest.

\section{References}

1. De Angelis R, Sant M, Coleman MP, et al.; EUROCARE-5 Working Group. Cancer survival in Europe 1999-2007 by country and age: results of EUROCARE-5 - a population-based study. Lancet Oncol 2014; 15: 23-34.
2. Denlinger CS, Carlson RW, Are M, et al. Survivorship: introduction and definition. Clinical practice guidelines in oncology. J Natl Compr Canc Netw 2014; 12: 34-45.

3. Bober SL, Varela VS. Sexuality in adult cancer survivors: challenges and interventions. J Clin Oncol 2012; 30: 3712-3719.

4. Engel GL. The need for a new medical model: a challenge for biomedicine. Science 1977; 196: 129-136.

5. Ussher JM, Perz J, Gilbert E. Changes to sexual well-being and intimacy after breast cancer. Cancer Nurs 2012; 35: 456-465.

6. Romito F, Goldzweig G, Cormio C, et al. Informal caregiving for cancer patients. Cancer 2013; 119 Suppl 11: 2160-2169.

7. Boszormenyi-Nagy I, Spark GM. Invisible Loyalties: Reciprocity in Intergenerational Family Therapy. Harper \& Row, New York 1973.

8. Andolfi M. La crisi della coppia: una prospettiva sistemico relazionale. Raffaello Cortina, Milano 1999.

9. Carter J, Goldfrank D, Schover LR. Simple strategies for vaginal health promotion in cancer survivors. J Sex Med 2011; 8: 549-559.

10. Katz A. The sounds of silence: sexuality information for cancer patients. J Clin Oncol 2005; 23: 238-241.

11. http://www.ascopost.com/issues/november-15,-2014/addressing-patients\%E2\%80\%99-sexual-dysfunction-throughout-survivorship.aspx; access: 1.12.2015.

12. Linkeviciute A, Boniolo G, Chiavari L, Peccatori FA. Fertility preservation in cancer patients: the global framework. Cancer Treat Rev 2014; 40: 1019-1027.

13. Horne G, Atkinson AD, Pease EH, et al. Live birth with sperm cryopreserved for 21 years prior to cancer treatment: case report. Hum Reprod 2004; 19: 1448-1449.

14. Shah DK, Goldman E, Fisseha S. Medical, ethical, and legal considerations in fertility preservation. Int J Gynaecol Obstet 2011; 115: 11-15.

15. Noyes N, Porcu E, Borini A. Over 900 oocyte cryopreservation babies born with no apparent increase in congenital anomalies. Reprod Biomed Online 2009; 18: 769-776.

16. Italian Ministry of Health. "Tavolo consultivo in materia di tutela e conoscenza della fertilità e prevenzione delle cause di infertilità" per il piano nazionale per la fertilità; available at: http://www.quotidianosanita.it/governo-e-parlamento/articolo.php?approfondimento_id=6290; access 1.12.2015.

17. Armuand GM, Rodriguez-Wallberg KA, Wettergren L, et al. Sex differences in fertility-related information received by young adult cancer survivors. J Clin Oncol 2012; 30: 2147-2153.

18. Geue K, Richter D, Schmidt R, et al. The desire for children and fertility issues among young German cancer survivors. J Adolesc Health 2014; 54: 527-535.

19. Hohmann C, Borgmann-Staudt A, Rendtorff R, et al. Patient counselling on the risk of infertility and its impact on childhood cancer survivors: results from a national survey. J Psychosoc Oncol 2011; 29: 274-285.

20. Gonçalves V, Sehovic I, Quinn G. Childbearing attitudes and decisions of young breast cancer survivors: a systematic review. Hum Reprod Update 2014; 20: 279-292.

21. Observatory on care conditions of cancer patients in Italy. Sixth report on care conditions of cancer patients. Roma: 2014; available at: www.favo.it/sesto-raporto.html; access 1.12.2015. 